\title{
Principais erros na fase pré-analítica de exames laboratoriais: uma revisão
}

\section{bibliográfica integrativa}

\author{
Main errors in the pre-analytical phase of laboratory tests: an integrative literature review \\ Principales errores en la fase preanalítica de las pruebas de laboratorio: una revisión integradora
} de la literatura

Ana Claudia Nascimento Sousa

ORCID: https://orcid.org/0000-0002-0532-6750 Universidade Nilton Lins, Brasil

E-mail: claudianascimento1037@gmail.com

Omero Martins Rodrigues Junior

ORCID: https://orcid.org/0000-0002-8552-3278 Universidade Nilton Lins, Brasil

E-mail: omeromartins.farm@gmail.com

\begin{abstract}
Resumo
Em um visão panorâmica dos serviços de diagnóstico promovidos pelos laboratórios, percebe-se que há uma relação de praticidade no auxílio do profissional da saúde a fim de elucidar o tratamento terapêutico aplicado no paciente, sendo necessário obedecer a legislação cabível para padronizar e proporcionar um controle de qualidade plausível dos resultados obtidos. A fase pré-analítica é a mais importante no processo de teste e envolve variáveis que não estão sob o controle do laboratório. Muito cuidado precisa ser tomado para examinar profundamente esta etapa da análise e manter um registro dos erros que surgem nela. Esta pesquisa tem como objetivo realizar um estudo sobre os principais erros da fase pré-analítica, enfatizando a coleta de materiais e de transporte, onde são encontrados os erros mais comuns, evidentes do processo no qual envolve em sua maioria, o trabalho manual. Desse modo, pode-se concluir que, a prática de manter um registro dos erros em todos os estágios da análise e, em seguida, desenvolver estratégias corretivas para sua prevenção pode libertar exponencialmente um laboratório de tais erros.
\end{abstract}

Palavras-chave: Erros; Pré-analítica; Laboratório; Diagnóstico.

\begin{abstract}
In a panoramic view of the diagnostic services provided by the laboratories, it is clear that there is a practical relationship in helping the health professional in order to elucidate the therapeutic treatment applied to the patient, being necessary to comply with the applicable legislation to standardize and provide control plausible quality of the results obtained. The pre-analytical phase is the most important in the testing process and involves variables that are not under the laboratory's control. Great care needs to be taken to look deeply into this step of the analysis and keep a record of the errors that arise in it. This research aims to carry out a study of the main errors of the pre-analytical phase, emphasizing the collection of materials and transport, where the most common errors are found, evident in the process in which it involves mostly manual work. Thus, it can be concluded that the practice of keeping a record of errors at all stages of the analysis and then developing corrective strategies for their prevention can exponentially free a laboratory of such errors.
\end{abstract}

Keywords: Errors; Pre-analytics; Laboratory; Diagnosis

\section{Resumen}

En una vista panorámica de los servicios de diagnóstico que brindan los laboratorios, se evidencia que existe una relación práctica en ayudar al profesional de la salud a dilucidar el tratamiento terapéutico aplicado al paciente, siendo necesario cumplir con la legislación aplicable para estandarizar y Proporcionar un control plausible de la calidad de los resultados obtenidos. La fase preanalítica es la más importante en el proceso de prueba e involucra variables que no están bajo el control del laboratorio. Se debe tener mucho cuidado para examinar en profundidad este paso del análisis y mantener un registro de los errores que surjan en él. Esta investigación tiene como objetivo realizar un estudio de los principales errores de la fase preanalítica, haciendo énfasis en la recolección de materiales y transporte, donde se encuentran los errores más comunes, evidentes en el proceso en el que implica principalmente trabajo manual. Así, se puede concluir que la práctica de llevar un registro de errores en todas las etapas del análisis y luego desarrollar estrategias correctivas para su prevención puede liberar exponencialmente a un laboratorio de tales errores.

Palabras clave: Errores; Preanalítica; Laboratorio; Diagnóstico. 


\section{Introdução}

De acordo com Andriolo et al. (2018), o diagnóstico laboratorial é um campo de rápido crescimento, que fornece uma contribuição significativa para a tomada de decisão clínica, apoiando a prevenção, o diagnóstico e o monitoramento terapêutico da maioria, senão de todas as patologias humanas. Nesse caso, melhoria e segurança em testes diagnósticos são essenciais para promover a meta de alta qualidade e biossegurança do processo de saúde de forma integral.

Na realidade, na concepção de Lima et al. (2017) e Lee (2019), todo o processo de teste laboratorial de um paciente, desde o pedido até o teste, seguida pela produção do relatório até, finalmente, o recebimento da assistência médica, pode ser dividido em três grandes etapas: pré-analítica (onde ocorre a coleta, o transporte e o processamento das espécies), analítica (a realização do teste) e pós-analítica (onde ocorre a leitura dos resultados, a interpretação e a realização dos retestes, caso necessite).

De um modo geral, os três processos combinados são conhecidos como processos de teste total. Nesse sentido, erros podem surgir em qualquer etapa e levar a produção de um relatório defeituoso que pode afetar o atendimento ao paciente como diagnóstico incorreto, tratamento inadequado, transfusão de sangue incompatível e assim por diante, erros que podem levar a graves consequências, possivelmente até a morte; é importante definir que erros podem ser quaisquer falhas ou defeitos, desde a solicitação de testes até o relatório dos resultados e a interpretação e reação adequadas sobre eles (Souza et al., 2021).

Dentre os erros pertinentes à fase pré-analítica tem-se: ordem de análise incorreta, ordem errada do teste marcado, solicitação incorreta do teste, solicitação de teste perdida, identificação errada do paciente, preparação errada do paciente, desconhecimento do tubo de coleta, erro na coleta da amostra, manuseio incorreto das amostras e transporte errado (Cezar, 2016; Tadese et al., 2018). Já na fase analítica, segundo Lima et al. (2015) e Saramela \& Fernandes (2021), têm-se os seguintes erros: amostra perdida, amostra misturada, equipamento quebrado e erro analítico. Para a fase pós-analítica têm-se: resultado de teste perdido, tempo de resposta, erro de transcrição e interpretação incorreta (Silva, Molin \& Mendes, 2016; Lima et al, 2017).

Para Feres et al. (2015), o laboratório de análises clínicas exerce um papel fundamental na promoção da saúde, pois auxilia nas decisões diagnósticas e terapêuticas, por intermédio da emissão do laudo, sendo este o documento que apresenta os resultados das análises laboratoriais, validados e autorizados pelo responsável técnico, sendo de grande importância a cautela na divulgação desses dados.

Dentro dessa perspectiva, os laboratórios devem cumprir com as normativas que visam a redução de erros, realizando programas com a finalidade de evitar falhas e resultados de impacto relevante. Dessa forma, os trabalhadores envolvidos com os laboratórios clínicos devem possuir conhecimento necessário para efetuar todas as normas que evitem futuras falhas e não consigam interferir no diagnóstico por meio de resultados falsos-positivos, indicando alguma patologia quando não há, ou falsos negativos, escondendo a doença presente; estima-se que cerca de 77\% dos erros que produzem resultados não consistentes com o quadro clínico do paciente são produzidos na fase pré-analítica (Oliveira \& Fernandes, 2016; Andriolo et al, 2018; Lee, 2019).

Por esse motivo, mesmo com a evolução gradual do processo de automação dos laboratórios clínicos, não se pode substituir o papel dos coletores, da etapa de coleta de material a ser analisado. Mediante a isso, é extremamente importante que todos os proponentes envolvidos nesse processo estejam conscientes de sua função e relevância para se obter um exame com alta qualidade, tendo como definição de qualidade a garantia de qualquer etapa realizada de forma correta, que possa oportunizar uma tomada de decisão médica e um atendimento eficaz ao paciente.

$\mathrm{Na}$ verdade, com o passar dos anos, ocorreram transformações que impactaram o cenário da saúde, influenciando diretamente na qualidade, na prestação de serviços laboratoriais e nas técnicas analíticas utilizadas. Souza et al (2021) declara que nas últimas décadas houve uma diminuição de dez vezes na taxa de erro analítico, devido ao surgimento de melhorias na confiabilidade e padronização da análise, novidades de técnicas, reagentes, instrumentação, avanços em tecnologia da informação, controle de qualidade e métodos de garantia de qualidade. 
No entanto, mesmo com todo esse avanço no quesito qualidade, eficiência e desempenho laboratorial, evidências indicam que a maioria das falhas acusadas no processo estão fora da fase analítica, e as etapas pré e pós-analíticas acabam sendo consideradas como as mais vulneráveis aos riscos e erros reais e aparentes. Nesse caso, a falta de atenção aos fatores extralaboratoriais está, portanto, em total contraste com o corpo de evidências que aponta para a multiplicidade de erros que continuam a ocorrer na fase pré-analítica.

Este estudo justifica-se pela relevância do tema em meio a escassez de trabalhos referentes a essa temática, sendo fundamental para o acadêmico da área de farmácia estar consciente de novos parâmetros e métodos analíticos a fim de valorizar a qualidade da realização dos testes laboratoriais, favorecendo a sociedade como um todo. A falta de estudos sobre o tema combinada ao fato de que grande parte das falhas em exames laboratoriais são relativas à fase pré-analítica expõe que esse campo pode ter grande impacto na realidade da medicina laboratorial, somando esforços em função do aumento da precisão dos exames por outras vias que não as mais recorrentemente abordagens sobre a análise laboratorial.

Desse modo, esta pesquisa tem como objetivo realizar um estudo sobre os principais erros da fase pré-analítica que podem impactar negativamente na precisão das leituras feitas no momento da análise, enfatizando a coleta de materiais e de transporte, onde são encontrados os erros mais comuns, evidentes do estágio do processo no qual envolve em sua maioria, o trabalho manual, propondo melhorias como sugestão para implementação a fim de evitar falhas tratáveis e solucionáveis.

\section{Metodologia}

De acordo com Marconi e Lakatos (2017), em fundamentos da metodologia científica, a metodologia de um trabalho científico pode ser definida como sendo uma combinação de normas básicas que precisam ser executadas na produção de conhecimento, com a finalidade de promover a ciência, isto é, um método utilizado para a busca de comprovação e pesquisas sobre determinado tema. A observação é o ponto de partida para todo método científico, utilizando-se de fatos, experimentos, indução e comprovação científica dos resultados obtidos. Isso confirma a ideia de que o método científico é um trabalho sistemático, na procura por respostas às questões estudadas, sendo este o caminho que se deve seguir para levar à formulação de uma teoria científica. É um estudo minucioso, que segue um percurso sistemático.

Esta pesquisa trata-se de uma revisão bibliográfica integrativa, em que a "metodologia utilizada [...] permite analisar estudos experimentais ou não, combinando literaturas empíricas e teóricas para discutir os fenômenos em sua complexidade real" (Mustafa et al, 2021, p.2) por intermédio de uma abordagem qualitativa, onde foram consultadas fontes secundárias a fim de identificar os principais erros da fase pré-analítica, principalmente no relativo à coleta de materiais e transporte, onde foram encontrados os erros mais comuns.

Trata-se de uma revisão de literatura, onde foram usadas as seguintes plataformas: Scientific Electronic Library Online - SCIELO, Público/editora MEDLINE - PUBMED, Google Acadêmico e Ministério da Saúde, utilizando-se de forma associada os descritores: "Erros, "Pré-analítica", "Laboratório Clínico", "Diagnóstico" e "Profissional Farmacêutico", onde o período de busca foi realizado nos meses de agosto a outubro/2021.

Foram contemplados artigos, legislações e dissertações nas plataformas já mencionadas, dentro do tempo estipulado de 6 anos (2015 a 2021), utilizando - se obras em idiomas como: português, inglês e espanhol. Os critérios de inclusão e exclusão serão por data, obras de acesso livre e títulos relacionados ao tema

\section{Resultados e Discussão}

Por meio dos resultados obtidos na literatura (Lima et al, 2017; Dhotre et al, 2020; Lee, 2019; Souza et al, 2021), pôdese observar que a maioria dos erros que afetam os resultados dos testes de laboratório ocorrem na fase pré-analítica (46-68\% do total de erros), podendo também ser encontrada uma elevada taxa de erros (19-47\% do total de erros) na fase pós-analítica. 
Segundo Souza et al (2021), os erros pré-analíticos são responsáveis por cerca de 70\% de todas as falhas provenientes dos diagnósticos de laboratório, onde a maioria é relativa a problemas relacionados à preparação do paciente, coleta de amostra, transporte e preparação para análise e armazenamento. Durante a preparação do paciente e coleta de amostra (incluindo a identificação do paciente e da amostra e manuseio da amostra) observa-se fontes frequentes de erros, onde se faz necessário uma maior cautela no transporte da amostra. Esta fase necessita de melhorias, visto que existe uma tendência crescente de consolidação das instalações laboratoriais, com a consequente necessidade de transporte de amostras a longa distância.

É importante ressaltar que, embora a fase pré-analítica seja conhecida por ser sujeita a erros, somente a pouco tempo os dados foram coletados para demonstrar que os erros ocorridos estão relacionados principalmente a procedimentos realizados fora das paredes do laboratório, por profissionais de saúde que não estão sob o controle direto da clínica laboratorial (Lee, 2019).

Nessa perspectiva, para que haja melhoria durante o processo de qualidade, deve-se levar em consideração a fase préanalítica clássica, ou seja, aquela parte que não foi realizada no laboratório clínico, muito menos manuseado pelos proponentes envolvidos no laboratório. Por essa razão, verifica-se que é primordial essa iniciativa pois prova que a automação de processos pré-analíticos repetitivos, propensos a erros e biosseguros realizados dentro das paredes do laboratório, diminuiu efetivamente os erros na preparação de amostras, centrifugação, preparação de alíquotas, pipetagem e classificação

No demais, Gil, Franco \& Galban (2016), acreditam que a fase pré-analítica é aquela que se inicia em ordem, a partir da solicitação do médico, incluindo a requisição de exame, preparação do paciente, coleta da amostra primária e transporte para e dentro do laboratório e terminando quando o procedimento de exame analítico é concluído. Isso reconhece claramente a necessidade de avaliar, monitorar e melhorar todos os procedimentos e processos na fase inicial do ciclo analítico.

Outro fator a ser discutido e avaliado é o indicador de qualidade confiável (QIs), essencial para permitir que os usuários quantifiquem a qualidade de um aspecto selecionado do atendimento, comparando-o com um critério definido. Para Dhotre et al (2020), um indicador de qualidade é, portanto, uma medida objetiva que avalia potencialmente todos os domínios de cuidados críticos, conforme definido pelo IOM (segurança do paciente, eficácia, equidade, foco no paciente, oportunidade e eficiência), que se baseia em evidências associadas a esses domínios, e pode ser implementado de maneira consistente e comparável em todas as configurações e ao longo do tempo.

Em uma visão panorâmica dos dados obtidos pelas literaturas estudadas (Santos \& Zanusso, 2015; Oliveira \& Fernandes, 2016; Cezar, 2016; Lima et al, 2017; Souza et al, 2021) verificou-se que os QIs devem ser elucidados para cobrir todas as etapas da fase pré-analítica, incluindo a adequação da seleção do teste, que é uma questão-chave em projetos que visam garantir a eficácia clínica. Nesse sentido, os indicadores de qualidade para a fase mais abrangente de erros (fase pré-analítica) devem ser identificados para sua utilização na preventiva e na busca pela qualidade perfeita do teste clínico, conforme a designação abaixo (Quadro 1). 
Quadro 1. Indicadores de qualidade na fase pré-analítica.

\begin{tabular}{|c|l|}
\hline Número do QIs & Indicadores de qualidade \\
\hline 1 & Adequação da solicitação de teste número de solicitações com pergunta clínica (\%) \\
\hline 2 & $\begin{array}{l}\text { Adequação da solicitação de teste Número de testes apropriados em relação à questão clínica } \\
(\%)\end{array}$ \\
\hline 3 & Número de requisição de exame de solicitações sem identificação do médico (\%) \\
\hline 4 & Número de requisição de exame de solicitações ininteligíveis (\%) \\
\hline 5 & Número de identificação de solicitações com identificação incorreta do paciente (\%) \\
\hline 6 & Número de identificação de solicitações com identificação incorreta do médico (\%) \\
\hline 7 & Número de pedidos de teste de pedidos com erros relativos à entrada de teste (\%) \\
\hline 8 & Amostras Número de amostras perdidas / não recebidas (\%) \\
\hline 9 & Amostras Número de amostras coletadas em recipientes inadequados (\%) \\
\hline 10 & Amostras Número de amostras hemolisadas (hematologia, química) (\%) \\
\hline 11 & Amostras Número de amostras coaguladas (hematologia, química) (\%) \\
\hline 12 & Amostras Número de amostras com volumes insuficientes (\%) \\
\hline 13 & Amostras Número de amostras com proporção inadequada de amostra-anticoagulante (\%) \\
\hline 14 & Amostras Número de amostras danificadas no transporte (\%) \\
\hline 15 & Número de amostras de amostras indevidamente rotuladas e armazenadas incorretamente (\%) \\
\hline
\end{tabular}

Fonte: Adaptada de Dhotre et al (2020)

Por meio desta tabela de indicadores de qualidade na fase pré-analítica verifica-se que, o desenvolvimento de QIs em programas de acreditação para medicina laboratorial é uma etapa fundamental para fornecer evidências sólidas de qualidade em todos os procedimentos e processos que envolvam as demandas dos laboratórios clínicos.

Dessa maneira, os indicadores de qualidade acabam por desempenhar desempenham uma função fundamental para garantir que atividades de melhoria contínua direcionadas com o objetivo de reduzir o risco de erros na prática clínica sejam realizadas. Entretanto, particularmente na fase pré-analítica (que investiga procedimentos que geralmente são realizados por operadores de saúde fora das paredes do laboratório), a coleta e monitoramento de dados sobre QIs, não resulta automaticamente em melhorias da qualidade, conforme ressalta Souza et al. (2021).

Lee (2019), enfatiza que as melhorias efetivas no início (e término) das etapas do processo laboratorial podem ser alcançadas apenas se esforços adicionais forem feitos para alcançar consenso sobre a preparação, adoção e monitoramento de procedimentos operacionais padrão eficazes nas etapas iniciais de testes de laboratório. Nesse cenário, em meio a pesquisa realizada, para o cumprimento dos objetivos deste estudo, sintetizamos como diversos autores mobilizam pontos semelhantes ao abordarem o tema dos erros pré-análise.

R. Silva e Lessa (2021) discutem a importância de um setor de controle de qualidade forte e perene dentro dos laboratórios para aumentar os cuidados referentes aos processos pré-analíticos de exames laboratoriais. Caberia a estes garantir o funcionamento da instituição de acordo com as boas práticas da pré-análise: instruir corretamente os pacientes com o que é necessário para a coleta da amostra e como fazê-lo; garantir uma estrutura suficiente para os profissionais coletarem, transportarem e controlarem as amostras corretamente e, ainda incluso nisso, treinar esses profissionais para que as chances de erro humano sejam ainda mais improvável.

Souza et al (2021) chegam à conclusão de que os erros pré-analíticos mais frequentes encontrados dentro de sua pesquisa são a hemólise, a quantidade insuficiente de material de amostra e a amostra coagulada, dando ênfase então à erros que ocorrem na coleta e na conservação do material até o laboratório, embora o artigo seja descritivo e não exponha uma solução nesse fenômeno.

Oliveira e Fernandes (2016) fazem um levantamento dos principais erros pré-analíticos que ocorreram em um laboratório privado na cidade de Maringá. Analisando os registros de aproximadamente dois anos (um ano e onze meses) viram que foram pedidos no total 707,449 coletas para exame, sendo que 0,18\% dessas coletas, 1274 delas, apresentaram-se 
insatisfatórias. Dessas amostras insatisfatórias 58,54\% tinham volume insuficiente, 19,29\% tinham amostras coaguladas, 9,43\% tinham hemólise, $8,21 \%$ foram consequência de preenchimento errado da identificação de amostra, 3\% das amostras foram lipêmicas e 1,56\% são apresentadas com impróprias sem maiores detalhes. Muitas dessas condições estão ligadas à conscientização do paciente, frente ao que é uma amostra razoável dentro dos padrões necessários para o exame, e outras relativas à organização, atenção e capacitação de pessoal da saúde, desde quem faz a coleta passando por quem faz o transporte até chegar em quem faz a triagem das amostras.

Teixeira et al (2016) mostram em seu texto sobre quais as principais causas de erros em pré-análises, análises e pósanálises a partir do acervo de pedidos de um laboratório público. Nos interessando, dado o escopo do artigo presente, aqueles listados na pré-análise, são citados a hemólise das amostras e as amostras em volume insuficiente para análise, o que denota por um lado erro na coleta e, por outro, falta de instrução do paciente.

Dando um passo a mais, Gil, Franco e Galban (2016), em estudo sobre os erros pré-analíticos de exames ocorridos em um hospital argentino, constatam que de 9141 casos de erros encontrados neste 82\% apresentaram pelo menos um erro préanalítico; ou seja, 7496 erros de pré-análise. Dessa forma, os autores destacam a importância da conscientização e do treinamento rigoroso dos profissionais que vão lidar com estes procedimentos.

Lee (2019), por outro lado, discute como apesar da variedade de erros possíveis em decorrência da complexidade inerente ao ambiente laboratorial-hospitalar, a maior parte dos achados de sua pesquisa eram relativos a erros na coleta, criando amostras insatisfatórias. Testa então uma intervenção nos serviços treinando equipes de médicos e enfermeiros sobre boas práticas na coleta e transporte de material em dois cursos rápidos de um dia, sendo que após esse treino viu a diminuição de 928 amostras insatisfatórias em 2018 para 771 em 2019.

Seguindo o raciocínio, Lima-Oliveira et al (2017) mostra como, dada a extensa variedade de possíveis erros entre a coleta de material do paciente até que esse chegue ao seu destino sob as lentes de um microscópio ou na pipeta para uma análise molecular, é necessário que haja um rigoroso controle sobre o treinamento do pessoal que fará desde a coleta até o transporte e da infraestrutura, uma vez que uma boa estrutura colabora para a conservação dos materiais enquanto uma má aumentará as chances de ocorrência dos erros pré-analíticos. Essa perspectiva já não é uma hipótese, mas sim uma explicação para o fenômeno observado em pesquisa anterior exposta em Lima-Oliveira et al (2015) sobre as causas de recalls de exames por parte dos laboratórios.

Dothre et al (2020) vão exatamente na mesma direção ao buscarem compreender o impacto do treinamento do pessoal encarregado com as diferentes tarefas dentro dos trabalhos necessários no momento pré-análise. Constatam, então, que se em um período de controle para o estudo, antes de treinar os diferentes trabalhadores que lidavam com a fase pré-analítica, 75,5\% dos erros encontravam-se nessa fase, após treiná-los essa taxa cai para apenas 24,5\%. Atestam então que um treinamento adequado pode ter alto impacto na qualidade dos exames e no aproveitamento das amostras coletadas.

Paterno (2019) faz uma abordagem semelhante, mas enfatizando exatamente a gravidade que a perda de tempo causada por erros pré-análise pode ter: estudando esse tema em relação a exames de detecção da tuberculose, a autora mostra como o tempo que se gasta entre a coleta de material, a impossibilidade de análise pelo caráter insatisfatória deste mesmo, a necessidade de pedir uma nova coleta e chegar a analisá-la é muito perigoso. Esse fato pode ser a diferença entre uma cura simples ou um quadro agravado que necessita de um tratamento que desgasta mais o paciente e gasta mais recursos da saúde público, sendo que isso seria evitável.

De acordo com toda essa bibliografia, pode-se constatar que os erros pré-analíticos nunca devem ser considerados inevitáveis, mas estes podem ser facilmente evitados com o treinamento correto e o uso de procedimentos de controle de qualidade adequados em todas as fases do processo de coleta e teste. Todos os proponentes da saúde devem ser obrigados a fazer aulas de educação continuada para garantir que não apenas estejam familiarizados com os procedimentos atuais, mas também 
que tomem conhecimento de quaisquer alterações que possam servir para reduzir o risco de ocorrência desse tipo de erro, e cabe aos laboratórios garantir acesso aos seus funcionários tanto desse treinamento quanto dessa estrutura de controle de qualidade.

\section{Conclusão}

Por meio desta pesquisa pôde-se inferir que, a dependência de resultados laboratoriais precisos para diagnósticos faz com que os laboratórios garantam a responsabilidade e a precisão dos resultados para negar o diagnóstico incorreto como consequência de relatórios com erros. A prática de manter um registro dos erros em todos os estágios da análise e, em seguida, desenvolver estratégias corretivas para sua prevenção pode libertar gradualmente um laboratório de tais erros.

Em uma visão panorâmica dos dados obtidos verificou-se que o conceito de gestão da qualidade total abrange todas as etapas envolvidas no processamento da amostra, desde a solicitação do teste até a interpretação final dos resultados pelos médicos para reduzir ou eliminar os erros que podem surgir durante as várias etapas.

Nesse sentido, a promoção de práticas ideais de coleta e procedimentos de transporte de amostras é um pré-requisito para a eficácia do funcionamento do laboratório, onde a dependência de resultados laboratoriais precisos para diagnósticos torna obrigatório que os laboratórios garantam a responsabilidade e a precisão dos resultados para negar o diagnóstico incorreto como consequência de relatórios com falhas. Esse processo precisa ser apoiado no treinamento adequado do pessoal envolvido nos processos pré-analíticos e na disponibilização de estrutura adequada por parte dos laboratórios.

Desse modo, a fase pré-analítica envolve fatores que devem ser levados em conta antes da realização do exame propriamente dito e que exercem influência direta na interpretação dos resultados. O exame reque uma preparação e orientação ao paciente, coleta da amostra, identificação, triagem, transporte e armazenamento, sendo que o não cumprimento de qualquer etapa desta fase gera não só dano ao paciente com o comparecimento para nova coleta, como compromete a credibilidade do laboratório junto a equipe médica, sendo de extrema importância a identificação das falhas evitáveis e a implementação de melhorias nesta primeira etapa de realização dos exames.

Logo, esta pesquisa vem a ser essencial ao oferecer bases sólidas sobre a temática, bem como favorecer a elucidação de novos modelos e métodos para a obtenção de respostas plausíveis para a problemática dos erros decorrentes na fase pré-analítica, auxiliando o acadêmico de farmácia na propagação de sua atuação profissional.

\section{Referências}

Andriolo, A., et al. (2018). Recomendações da sociedade brasileira de patologia clínica/medicina laboratorial (SBPC/ML): fatores pré-analíticoss e interferentes em ensaios laboratorias. Editora Manole Ltda.

Cezar, F. M. (2016). Controle de qualidade laboratorial: uma atualização de urinálise (Trabalho de conclusão de curso). Centro de ciências farmacêuticas, Universidade Federal do Paraná

Dhotre, P. S., Dhore, S. V. \& Shaikh, A. K. A. R. (2020). Comparative study of pre-analytical erros in central clinical laboratory in a tertiary care hospital in Maharashtra. Journal of Krishna Institute of Medical Sciences University, 9(2), 67-72.

Féres, V. C de R., Lopes, F. M., Rocha, B. A. M. \& Alcanfor, J. D. X. (2015). Avaliação de indicadores laboratoriais no Laboratório Escola da Faculdade de Farmácia - UFG. Revista Vita Sanitas, 9(2), 10-23.

Gil, A. C. (2017). Como elaborar projetos de pesquisa. (6ed) São Paulo: Atlas.

Gil, P., Franco, M. \& Galbán, G. (2016). Evaluación de errores preanalíticos em el laboratório de planta del HIGA O. Alende de Mar del Plata. Acta Bioquimica clínica latinoam, 50(30), 463-468.

Lee, N. Y. (2019). Reduction of pre-analytical errors in the clinical laboratory at the University Hospital of Korea through quality improvement activities. Clinical Biochemistry, 70, 24-29.

Lima-Oliveira, G, Lippi G, Salvagno G. L, Brocco G \& Guidi, G. C. (2015). In vitro diagnostic company recalls and medical laboratory practices: an Italian case. Biochem Med 25(2), 273-278.

Lima-Oliveira, G., Volanski, W., Lippi, G., Picheth, G. \& Guidi, G.C. (2017). Preanalytical phase management: a review of the procedures from patient preparation to laboratory analysis. Scand J Clin Lab Invest, 77, $153-163$. 
Marcone, M. A. \& Lakatos, E. M. (2017). Fundamentos de Metodologia Científica. (8 ed) São Paulo: Atlas.

Mustafa, M. M., Souza, E. P. P., Sena, A. B. (2021). Menopausa precoce no Brasil: uma revisão bibliográfica integrativa. Research, Society and Development, $10(14), 1-8$

Oliveira, C. F. \& Fernandes, T. R. L. (2016). Analysis of the pre-analytical phase in a private pathology laboratory of Maringá city-PR, Brazil. Jornal Brasileiro de Patologia e Medicina Laboratorial, 52(2), 78-83.

Paterno, K. C. (2019). Análise de erros pré-analíticos na rotina laboratorial de tuberculose. Araçatuba: Instituto Adolfo Lutz.

R. Silva, A., Lessa, L. H. Os principais erros da fase pré-analítica dos laboratórios de análises clínicas da região metropolitana de americana: percepção dos clientes. (2021). Revista de Trabalhos Acadêmicos da FAM: TCC 2020, 6(1), 64.

Santos, A.P. \& Zanusso Junior, G. (2015). Controle de qualidade em laboratórios clínicos. Revista Uningá, 45 , 60-67.

Saramela, M. M. \& Fernandes, T. R. L (2021). Avaliação da fase pré-analítica do exame de urina de rotina em laboratório privado da cidade de Maringá, Paraná, Brasil. J Bras Patol Med Lab, 57, 1-6.

Sousa, R. L., Sousa, D. S., Barbosa, M. C. de M., Silva, A. F., Resende, L. J., Brito, G. C., Nascimento Junior, J. A. A. \& Oliveira, T. V. L. (2021). Erros préanalíticos em laboratórios de análises clínicas: uma revisão. Brazilian Journal of Health Review, 4(2), 9132-9142.

Silva, B., Molin, D. B. D. \& Mendes, G. A. (2016). Adequabilidade de amostras de urina recebidas por um laboratório de análises clínicas do noroeste do estado do Rio Grande do Sul. Revista Bra Análises Clínicas, 48(4), 352-355.

Tadesse, H., Desta, K., Kinde, S., Hassen, F., Gize, A. (2018). Clinical chemistry laboratory erros at ST. Paul's Hospital Millennium Medical College (SPHMMC), Addis Abba, Ethiopia. BMC Research Notes, 11(1), 1-5.

Teixeira, J. C. C., Chicote, S. R. M. \& Daaneze, E. R. (2016). Não conformidades identificadas durante as fases pré-analítica, analítica e pós-análitica de um laboratório público de análises clínicas. Nucleus, 13(1), 251-560. 